Analele Universităţii de Vest, Timişoara

Seria Matematică - Informatică

LIV , 1, (2016), 35- 53

\title{
Deszcz Pseudo Symmetry Type LP-Sasakian Manifolds
}

Kanak Kanti Baishya and Partha Roy Chowdhury

\begin{abstract}
Recently the present authors introduced the notion of generalized quasi-conformal curvature tensor which bridges Conformal curvature tensor, Concircular curvature tensor, Projective curvature tensor and Conharmonic curvature tensor. This paper attempts to charectrize LP-Sasakian manifolds with $\omega(X, Y) \cdot \mathcal{W}=$ $L\left\{\left(X \wedge_{g} Y\right) \cdot \mathcal{W}\right\}$. On the basis of this curvature conditions and by taking into account, the permutation of different curvature tensors we obtained and tabled the nature of the Ricci tensor for the respective pseudo symmetry type LP-Sasakian manifolds.
\end{abstract}

AMS Subject Classification (2000). 53C15; 53C25.

Keywords. generalized quasi-conformal curvature tensor ; $\phi$ semi-symmetric; pseudo- symmetry type LP-Sasakian manifolds.

\section{Introduction}

Recently, in tune with Yano and Sawaki [19], the first authors [14] have introduced and studied generalized quasi-conformal curvature tensor $\mathcal{W}$ in the context of $N(k, \mu)$-manifold. The components of quasi-conformal like curvature tensor $\mathcal{W}$ in a Riemannian manifold $\left(M^{2 n+1}, g\right)(n>1)$, are given 
by

$$
\begin{aligned}
\mathcal{W}(X, Y) Z= & \frac{2 n-1}{2 n+1}[(1+2 n a-b)-\{1+2 n(a+b)\} c] C(X, Y) Z \\
& +[1-b+2 n a] E(X, Y) Z+2 n(b-a) P(X, Y) Z \\
& +\frac{2 n-1}{2 n+1}(c-1)\{1+2 n(a+b)\} \hat{C}(X, Y) Z
\end{aligned}
$$

where scalar triples $(a, b, c)$ are real constants and $C, \hat{C}, E, P$ stands for Conformal, Conharmonic, Concircular and Projective curvature tensors respectively for all $X, Y \& Z \in \chi(M)$, the set of all vector fields of the manifold $M$. The beauty of such curvature tensor lies in the fact that it has the flavour of Riemann curvature tensor $R$ if the scalar triple $(a, b, c) \equiv(0,0,0)$, Conformal curvature tensor $C[21]$ if $(a, b, c) \equiv\left(-\frac{1}{2 n-1},-\frac{1}{2 n-1}, 1\right)$, Conharmonic curvature tensor $\hat{C}[38]$ if $(a, b, c) \equiv\left(-\frac{1}{2 n-1},-\frac{1}{2 n-1}, 0\right)$, Concircular curvature tensor $E([18]$, p. 84$)$ if $(a, b, c) \equiv(0,0,1)$, Projective curvature tensor $P([18]$, p. 84$)$ if $(a, b, c) \equiv\left(-\frac{1}{4 n}, 0,0\right)$ and $m$-Projective curvature tensor $H$ [13], if $(a, b, c) \equiv\left(-\frac{1}{4 n},-\frac{1}{4 n}, 0\right)$. The equation $(1.1)$ can also be written as

$$
\begin{aligned}
& W(X, Y) Z \\
= & R(X, Y) Z+a[S(Y, Z) X-S(X, Z) Y]+b[g(Y, Z) Q X-g(X, Z) Q Y] \\
& -\frac{c r}{2 n+1}\left(\frac{1}{2 n}+a+b\right)[g(Y, Z) X-g(X, Z) Y] .
\end{aligned}
$$

$R, S, Q \& r$ being Christoffel Riemannian curvature tensor, Ricci tensor, Ricci operator and scalar curvature respectively.

In 1989 K. Matsumoto [16] introduced the notion of Lorentzian paraSasakian (LP-Sasakian for short) manifold. Mihai and Rosca [20] also defined the same notion independently. This type of manifold is also discussed in ([1], [2]) and the references therein. We refer to Section 2 for precise definitions of the symbols used.

An LP-Sasakian manifold $\left(M^{2 n+1}, g\right), n \geq 1$, is said to be pseudo-symmetry type according to Deszcz ([29], [32], [12], [11]) respectively Ricci pseudo symmetry type $([30],[31],[9])$ if

$$
\omega(X, Y) \cdot \mathcal{W}=L\left\{\left(X \wedge_{g} Y\right) \cdot \mathcal{W}\right\}\left(\operatorname{resp} \cdot \omega(X, Y) \cdot \mathcal{W}=L\left\{\left(X \wedge_{S} Y\right) \cdot \mathcal{W}\right\}\right),
$$

where $\omega$ and $\mathcal{W}$ stand for generalized quasi-conformal curvature tensor with the associated scalar triples $(\bar{a}, \bar{b}, \bar{c})$ and $(a, b, c)$ respectively (two distinct notions have been used in order to study the nature of 36 pseudo symmetric type curvature condition as shown in the table by taking into account 
the permutation and combination of the scalar triples), the dot means that $\omega(X, Y)$ acts as a derivation on $\mathcal{W}, L$ is a smooth function and the endomorphism field $X \wedge_{A} Y$ is defined by

$$
\left(X \wedge_{A} Y\right) Z=A(Y, Z) X-A(X, Z) Y
$$

for all vector fields $X, Y, Z$ on $M$ and it similarly acts as a derivation on $\mathcal{W}$. In particular, manifold satisfying the condition $R(X, Y) \cdot R=L\left\{\left(X \wedge_{g} Y\right) \cdot R\right\}$ (obtained from (1.3) by setting $\bar{a}=\bar{b}=\bar{c}=0=a=b=c$ in (1.3)) is said to be pseudo symmetric. If $L$ is constant, $M$ is called a pseudo symmetric manifold of constant type and if particularly $L=0$ then $M$ is called a semisymmetry type manifold for details (see [4], [5], [8], [17], [27], [39], [26], [40], [41] and the references therein). Semi-symmetric spaces are a generalization of locally symmetric spaces $(\nabla R=0),([6],[28])$ while pseudo symmetric spaces are a natural generalization of semi-symmetric spaces. Note that the Schwarzschild spacetime, the Kottler spacetime, the Reissner-Nordström spacetime, as well as some Friedmann- Lemaître- Robertson-Walker spacetimes are the "oldest" examples of non semi-symmetric pseudo symmetric warped product manifolds( [33], [35]).

Our work is structured as follows. Section 2 is a very brief account of LPSasakian manifolds. LP-Sasakian manifold with $\mathcal{W}(X, Y) \cdot \phi=0$ is studied in section 3 and it is found that such a manifold is an $\eta$-Einstein space provided $a \neq 0$. In section 4 , we paid our special attention to investigate pseudosymmetry type LP-Sasakian manifold( i.e., $\left.\omega(X, Y) \cdot \mathcal{W}=L\left\{\left(X \wedge_{g} Y\right) \cdot W\right\}\right)$. On the basis of this condition and by taking into account, the permutation of different curvature tensors we obtained and tabled the nature of the Ricci tensor for the respective pseudo-symmetry type LP-Sasakian manifold.

\section{$2 \quad$ LP-Sasakian manifolds}

An $n$-dimensional differentiable manifold $M$ is said to be an LP-Sasakian manifold ([37], [16])if it admits a $(1,1)$ tensor field $\phi$, a unit timelike contravariant vector field $\xi$, a 1-form $\eta$ and a Lorentzian metric $g$ which satisfy

$$
\begin{gathered}
\eta(\xi)=-1, \quad g(X, \xi)=\eta(X), \quad \phi^{2} X=X+\eta(X) \xi \\
g(\phi X, \phi Y)=g(X, Y)+\eta(X) \eta(Y), \quad \nabla_{X} \xi=\phi X \\
\left(\nabla_{X} \phi\right)(Y)=g(X, Y) \xi+\eta(Y) X+2 \eta(X) \eta(Y) \xi
\end{gathered}
$$


where $\nabla$ denotes the operator of covariant differentiation with respect to the Lorentzian metric $g$. It can be easily seen that in an LP-Sasakian manifold, the following relations hold :

$$
\phi \xi=0, \quad \eta(\phi X)=0, \quad \operatorname{Rank} \phi=n-1 .
$$

Again, if we put

$$
\Omega(X, Y)=g(X, \phi Y)
$$

for any vector fields $X, Y$ then the tensor field $\Omega(X, Y)$ is a symmetric $(0,2)$ tensor field ([20], [37]). Also, since the vector field $\eta$ is closed in an LPSasakian manifold, we have ([16], [20])

$$
\left(\nabla_{X} \eta\right)(Y)=\Omega(X, Y), \quad \Omega(X, \xi)=0
$$

for any vector fields $X$ and $Y$.

Let $M$ be an $n$-dimensional LP-Sasakian manifold with structure $(\phi, \xi, \eta, g)$. Then the following relations hold ([16], [20], [3]) :

$$
\begin{gathered}
g(R(X, Y) Z, \xi)=\eta(R(X, Y) Z)=g(Y, Z) \eta(X)-g(X, Z) \eta(Y), \\
R(\xi, X) Y=g(X, Y) \xi-\eta(Y) X \\
R(X, Y) \xi=\eta(Y) X-\eta(X) Y, \\
S(X, \xi)=2 n \eta(X), \\
S(\phi X, \phi Y)=S(X, Y)+2 n \eta(X) \eta(Y),
\end{gathered}
$$

for any vector fields $X, Y, Z$ where $R$ is the Riemannian curvature tensor of the manifold.

Lemma 2.1. Let $M^{2 n+1}(\phi, \xi, \eta, g)$ be an LP-Sasakian manifold. Then for any vector fields $X, Y, Z$ on $M^{2 n+1}$, the following relation holds:

$$
\begin{aligned}
& R(X, Y) \phi Z-\phi R(X, Y) Z \\
= & g(Y, Z) \phi X-g(X, Z) \phi Y+g(X, \phi Z) Y-g(Y, \phi Z) X \\
& +2\{g(X, \phi Z) \eta(Y)-g(Y, \phi Z) \eta(X)\} \\
& +2\{\eta(Y) \phi X-\eta(X) \phi Y\} \eta(Z) .
\end{aligned}
$$

Lemma 2.2. Let $\left(M^{2 n+1}, g\right)$ be an LP-Sasakian manifold. If $Q$ is the Ricci operator, i.e. , if $S(X, Y)=g(Q X, Y)$ for all vector fields $X, Y$ on $M^{2 n+1}$, then

$$
Q \phi=\phi Q
$$


Let $A, B$ be two symmetric $(0,2)$-tensors on $M$. Their Kulkarni-Nomizu product $A \wedge B$ is defined on $\chi(M)$ by:

$$
\begin{aligned}
(A \wedge B)(X, Y, Z, U) & =A(X, U) B(Y, Z)+A(Y, Z) B(X, U) \\
& -A(X, Z) B(Y, U)-A(Y, U) B(X, Z) .
\end{aligned}
$$

In particular, when $A=B=g$, we have the Kulkarni-Nomizu squared $g \wedge g$ [15]:

$$
(g \wedge g)(X, Y, Z, \mathcal{W})=2[g(X, \mathcal{W}) g(Y, Z)-g(X, Z) g(Y, \mathcal{W})]
$$

We notice that

$$
(g \wedge g)(X, Y, Z, \mathcal{W})=2 g\left(\left(X \wedge_{g} Y\right)(Z), \mathcal{W}\right)
$$

This leads to the $(0,4)$-tensor $G=\frac{1}{2}(g \wedge g)$; it is defined as follows:

$$
G(X, Y, Z, \mathcal{W})=g(X, \mathcal{W}) g(Y, Z)-g(X, Z) g(Y, \mathcal{W})
$$

\section{$3 \quad$ LP-Sasakian manifolds satisfying $W(\mathrm{X}, \mathrm{Y}) \cdot \phi=0$}

Definition 3.1. A $(2 n+1)$-dimensional $(n>1)$ LP-Sasakian manifold is said to be $\phi$-semi-symmetric if the generalized quasi-conformal curvature tensor obeys the condition $\mathcal{W}(X, Y) \cdot \phi=0$ for any vector fields $X, Y$ on the manifold.

Theorem 3.1. A $\phi$-semi-symmetric LP-Sasakian manifold $M^{2 n+1}$ is $\eta$-Einstein provided $a \neq 0$.

Proof. Let us consider an LP-Sasakian manifold admitting $\mathcal{W}(X, Y) \cdot \phi=0$.

$$
\text { i.e. } \mathcal{W}(X, Y) \phi Z-\phi \mathcal{W}(X, Y) Z=0 \text {. }
$$

In view of (1.2), we can easily bring out

$$
\begin{aligned}
& R(X, Y) \phi Z-\phi R(X, Y) Z+a[S(Y, \phi Z) X-S(X, \phi Z) Y \\
& -S(Y, Z) \phi X+S(X, Z) \phi Y]+b[g(Y, \phi Z) Q X-g(X, \phi Z) Q Y \\
& -g(Y, Z) \phi Q X+g(X, Z) \phi Q Y]-\frac{c r}{2 n+1}\left(\frac{1}{2 n}+a+b\right)[g(Y, \phi Z) X \\
& -g(X, \phi Z) Y-g(Y, Z) \phi X+g(X, Z) \phi Y]=0 .
\end{aligned}
$$


By virtue of Lemma (2.11) \& (2.12), equation (3.2) reduces to

$$
\begin{aligned}
& {[g(Y, Z) \phi X-g(X, Z) \phi Y+g(X, \phi Z) Y-g(Y, \phi Z) X+} \\
& 2\{g(X, \phi Z) \eta(Y)-g(Y, \phi Z) \eta(X)\}+2\{\eta(Y) \phi X-\eta(X) \phi Y\} \eta(Z)] \\
& +a[S(Y, \phi Z) X-S(X, \phi Z) Y-S(Y, Z) \phi X+S(X, Z) \phi Y] \\
& +b[g(Y, \phi Z) Q X-g(X, \phi Z) Q Y-g(Y, Z) Q \phi X+g(X, Z) Q \phi Y] \\
& -\frac{c r}{2 n+1}\left(\frac{1}{2 n}+a+b\right)[g(Y, \phi Z) X-g(X, \phi Z) Y-g(Y, Z) \phi X \\
& +g(X, Z) \phi Y]=0 .
\end{aligned}
$$

Replacing $X$ by $\phi X$, and then taking inner product with $U$, equation (3.3) becomes

$$
\begin{aligned}
& g(Y, Z) g(X, U)+\eta(X) \eta(U) g(Y, Z)-\Omega(X, Z) \Omega(Y, U)+g(X, Z) g(Y, U) \\
& +\eta(X) \eta(Z) g(Y, U)-\Omega(Y, Z) \Omega(X, U)+2 \eta(Y) \eta(U) g(X, Z) \\
& +2 \eta(X) \eta(Y) \eta(Z) \eta(U)+2 \eta(Y) \eta(Z) g(X, U)+2 \eta(X) \eta(Y) \eta(Z) \eta(U) \\
& +a[S(Y, \phi Z) \Omega(X, U)-S(X, Z) g(Y, U)-2 n \eta(X) \eta(Z) g(Y, U) \\
& -S(Y, Z) g(X, U)-S(Y, Z) \eta(X) \eta(U)+S(\phi X, Z) \Omega(Y, U)] \\
& +b[\Omega(Y, Z) S(\phi X, U)-g(X, Z) S(Y, U)-S(Y, U) \eta(X) \eta(Z) \\
& -g(Y, Z) S(X, U)-2 n \eta(X) \eta(U) g(Y, Z)+\Omega(X, Z) S(\phi Y, U)] \\
& -\frac{c r}{2 n+1}\left(\frac{1}{2 n}+a+b\right)[\Omega(Y, Z) \Omega(X, U)-g(X, Z) g(Y, U) \\
& -\eta(X) \eta(Z) g(Y, U)-g(Y, Z) g(X, U)-\eta(X) \eta(U) g(Y, Z) \\
& +\Omega(X, Z) \Omega(Y, U)]=0 .
\end{aligned}
$$

Replacing $Y$ and $U$ by $\xi$ in (3.4), we obtain

where $\quad a \neq 0$

$$
\begin{aligned}
S(X, Z)= & -\frac{1}{a}\left\{1+2 n b-\frac{c r}{2 n+1}\left(\frac{1}{2 n}+a+b\right)\right\} g(X, Z) \\
& -\frac{1}{a}\left\{1+2 n(a+b)-\frac{c r}{2 n+1}\left(\frac{1}{2 n}+a+b\right)\right\} \eta(X) \eta(Z),
\end{aligned}
$$

This completes the proof. 
Remark 3.1. The equation (3.5) is also true for an LP-Sasakian manifold $\left(M^{2 n+1}, g\right), n \geq 1$ with vanishing generalized quasi-conformal tensor.

\section{Pseudo symmetry type LP-Sasakian manifold}

Definition 4.1. ([10], [34]) An LP-Sasakian manifold $\left(M^{2 n+1}, g\right), n \geq 1$ is said to be pseudo symmetry type, if the generalized quasi-conformal tensor $\omega($ or $\mathcal{W})$ admits

$$
\begin{aligned}
& (\omega(X, Y) \cdot \mathcal{W})(Z, U) V)=L_{\omega}((X \wedge Y) \cdot \mathcal{W})(Z, U) V \\
& \omega(X, Y) \mathcal{W}(Z, U) V \\
= & \mathcal{W}(\omega(X, Y) Z, U) V+\mathcal{W}(Z, \omega(X, Y) U) V+\mathcal{W}(Z, U) \omega(X, Y) V \\
+ & L[\overline{\mathcal{W}}(Z, U, V, Y) X+\overline{\mathcal{W}}(Z, U, V, X) Y+g(Y, Z) \mathcal{W}(X, U) V \\
- & g(X, Z) \mathcal{W}(Y, U) V+g(Y, U) \mathcal{W}(Z, X) V-g(X, U) \mathcal{W}(Z, Y) V \\
+ & g(Y, V) \mathcal{W}(Z, U) X-g(X, V) \mathcal{W}(Z, U) Y] .
\end{aligned}
$$

where $\omega$ and $W$ stands for generalized quasi-conformal curvature tensor with associate scalars $(\bar{a}, \bar{b}, \bar{c})$ and $(a, b, c)$ respectively.

If $\omega \cdot \mathcal{W}=0$ then $M$ is called generalized quasi-conformally semi-symmetric. A pseudo-symmetric space is said to be proper if it is not semi-symmetric. For details we refer to ([7], [22]). In the literature, there is also another notion of pseudo-symmetry.

Definition 4.2. An LP-Sasakian manifold $(M, g)$ is said to be pseudo-symmetry type in the sense of Chaki [24] if

$$
\begin{aligned}
& \left(\nabla_{X} \mathcal{W}\right)(Y, Z, U, V) \\
= & 2 A(X) \mathcal{W}(Y, Z, U, V)+A(Y) \mathcal{W}(X, Z, U, V)+A(Z) \mathcal{W}(Y, X, U, V)+ \\
& A(U) \mathcal{W}(Y, Z, X, V)+A(V) \mathcal{W}(Y, Z, U, X),
\end{aligned}
$$

for all $Y, Z, U, V \in \chi(M)$, where $A$ is a non-zero1-form on $(M, g)$.

Of course, both the definitions of pseudo-symmetry type for a semi- Riemannian manifold are not equivalent. For example, in contact geometry, every Sasakian space form is pseudo-symmetric in the sense of Belkhelfa, Deszcz and Verstraelen ([23], Theorem 2.3, Corollary 2.4), but a Sasakian 
manifold cannot be pseudo-symmetric in the sense of Chaki ([25], Theorem 1 ). We assume the pseudo-symmetry always in the sense of Deszcz, unless specifically stated otherwise.

Let us consider a $(2 n+1)$-dimensional pseudo-symmetry type LP-Sasakian manifold. Then from the equation (4.2), we get

$$
\begin{aligned}
& g(\omega(\xi, X) \mathcal{W}(Y, Z) U, \xi)-g(\mathcal{W}(\omega(\xi, X) Y, Z) U, \xi) \\
& -g(\mathcal{W}(Y, \omega(\xi, X) Z) U, \xi)-g(\mathcal{W}(Y, Z) \omega(\xi, X) U, \xi) \\
= & L[-\overline{\mathcal{W}}(Y, Z, U, X)-\eta(\mathcal{W}(Y, Z) U) \eta(X)-g(X, Y) \eta(\mathcal{W}(\xi, Z) U) \\
+ & \eta(Y) \eta(\mathcal{W}(X, Z) U)-g(X, Z) \eta(\mathcal{W}(Y, \xi) U)+\eta(Z) \eta(\mathcal{W}(Y, X) U) \\
& +\eta(U) \eta(\mathcal{W}(Y, Z) X)] .
\end{aligned}
$$

Putting $X=Y=e_{i}$ in (4.4) where $\left\{e_{1}, e_{2}, e_{3}, \ldots, e_{2 n}, e_{2 n+1}=\xi\right\}$ is an orthonormal basis of the tangent space at each point of the manifold $M$ and taking the summation over $i, 1 \leq i \leq 2 n+1$, we get

$$
\begin{aligned}
& \sum_{i=1}^{2 n+1}\left[g\left(\omega\left(\xi, e_{i}\right) \mathcal{W}\left(e_{i}, Z\right) U, \xi\right)-g\left(\mathcal{W}\left(\omega\left(\xi, e_{i}\right) e_{i}, Z\right) U, \xi\right)\right. \\
& \left.-g\left(\mathcal{W}\left(e_{i}, \omega\left(\xi, e_{i}\right) Z\right) U, \xi\right)-g\left(\mathcal{W}\left(e_{i}, Z\right) \omega\left(\xi, e_{i}\right) U, \xi\right)\right] \\
= & L \sum_{i=1}^{2 n+1}\left[-\overline{\mathcal{W}}\left(e_{i}, Z, U, e_{i}\right)-\eta\left(\mathcal{W}\left(e_{i}, Z\right) U\right) \eta\left(e_{i}\right)-g\left(e_{i}, e_{i}\right) \eta(\mathcal{W}(\xi, Z) U)\right. \\
& +\eta\left(e_{i}\right) \eta\left(\mathcal{W}\left(e_{i}, Z\right) U\right)-g\left(e_{i}, Z\right) \eta\left(\mathcal{W}\left(e_{i}, \xi\right) U\right)+\eta(Z) \eta\left(\mathcal{W}\left(e_{i}, e_{i}\right) U\right) \\
& \left.+\eta(U) \eta\left(\mathcal{W}(e, Z) e_{i}\right)\right] .
\end{aligned}
$$

From the equation (1.2), we can easily bring out the followings

$$
\begin{aligned}
& \eta(\mathcal{W}(\xi, U) Z) \\
= & {\left[\frac{c r}{2 n+1}\left(\frac{1}{2 n}+a+b\right)-2 n a-2 n b-1\right] \eta(Z) \eta(U) } \\
& +\left[\frac{c r}{2 n+1}\left(\frac{1}{2 n}+a+b\right)-2 n b-1\right] g(Z, U)-a S(Z, U), \\
& \sum_{i=1}^{2 n+1} \overline{\mathcal{W}}\left(e_{i}, Z, U, e_{i}\right) \\
= & (1-b+2 n a) S(Z, U)+\left\{b r-\frac{2 n c r}{2 n+1}\left(\frac{1}{2 n}+a+b\right)\right\} g(Z, U),
\end{aligned}
$$




$$
\begin{aligned}
& \sum_{i=1}^{2 n+1} \eta\left(\mathcal{W}\left(e_{i}, Z\right) e_{i}\right) \\
= & {\left[-2 n(1-a+2 n b)-\left\{a r-\frac{2 n c r}{2 n+1}\left(\frac{1}{2 n}+a+b\right)\right\}\right] \eta(Z), } \\
& \sum_{i=1}^{2 n+1} S\left(\mathcal{W}\left(e_{i}, Z\right) U, e_{i}\right) \\
= & \left\{a r+\frac{c r}{2 n+1}\left(\frac{1}{2 n}+a+b\right)\right\} S(Z, U)-(a+b-1) S^{2}(Z, U) \\
& -\frac{1}{2 n+1}\left(\frac{1}{2 n}+a+b\right) g(Z, U)+b S\left(Q e_{i}, e_{i}\right) g(Z, U), \\
= & \sum_{i=1}^{2 n+1} \eta\left(e_{i}\right) \eta\left(\mathcal{W}\left(Q e_{i}, Z\right) U\right) \\
= & 2 n\left[1+2 n b-\frac{c r}{2 n+1}\left(\frac{1}{2 n}+a+b\right)\right] g(Z, U)-2 n a S(Z, U) \\
& +\left\{1+2 n\left[1+2 n(a+b)-\frac{c r}{2 n+1}\left(\frac{1}{2 n}+a+b\right)\right] \eta(Z) \eta(U),\right. \\
& \sum_{i=1}^{2 n+1} S\left(e_{i}, Z\right) \eta\left(\mathcal{W}\left(e_{i}, \xi\right) U\right) \\
& \left.2 n(a+b)-\frac{c r}{2 n+1}\left(\frac{1}{2 n}+a+b\right)\right] \eta(Z) \eta(U) \\
& \left.\left\{\frac{1}{2 n}+a+b\right)\right\} S(Z, U)+a S^{2}(Z, U) .(4.1 \\
& \{r
\end{aligned}
$$

Now

$$
\begin{aligned}
& \sum_{i=1}^{2 n+1} g\left(\omega\left(\xi, e_{i}\right) \mathcal{W}\left(e_{i}, Z\right) U, \xi\right) \\
= & {\left[\frac{\bar{c} r}{2 n+1}\left(\frac{1}{2 n}+\bar{a}+\bar{b}\right)-2 n \bar{b}-1\right]\left\{\overline{\mathcal{W}}\left(e_{i}, Z, U, e_{i}\right)+\eta\left(\mathcal{W}\left(e_{i}, Z\right) U\right) \eta\left(e_{i}\right)\right\} } \\
& -\bar{a}\left[S\left(\mathcal{W}\left(e_{i}, Z\right) U, e_{i}\right)+2 n \eta\left(\mathcal{W}\left(e_{i}, Z\right) U\right) \eta\left(e_{i}\right)\right]
\end{aligned}
$$




$$
\begin{aligned}
= & {\left[\frac{\bar{c} r}{2 n+1}\left(\frac{1}{2 n}+\bar{a}+\bar{b}\right)-2 n \bar{b}-1\right] \overline{\mathcal{W}}\left(e_{i}, Z, U, e_{i}\right)-\bar{a} S\left(\mathcal{W}\left(e_{i}, Z\right) U, e_{i}\right) } \\
& +\left[\frac{\bar{c} r}{2 n+1}\left(\frac{1}{2 n}+\bar{a}+\bar{b}\right)-2 n \bar{b}-1-2 n \bar{a}\right] \eta(\mathcal{W}(\xi, U) Z) .
\end{aligned}
$$

In view of (4.7) \& (4.9), (4.12) becomes

$$
\begin{aligned}
& g\left(\omega\left(\xi, e_{i}\right) \mathcal{W}\left(e_{i}, Z\right) U, \xi\right) \\
= & {\left[\left\{\frac{\bar{c} r}{2 n+1}\left(\frac{1}{2 n}+\bar{a}+\bar{b}\right)-2 n \bar{b}-1\right\}(1+2 n a-b)\right.} \\
& \left.-\bar{a}\left\{a r+\frac{c r}{2 n+1}\left(\frac{1}{2 n}+a+b\right)\right\}\right] S(Z, U)+\bar{a}(a+b-1) S^{2}(Z, U) \\
& +\left\{\frac{\bar{c} r}{2 n+1}\left(\frac{1}{2 n}+\bar{a}+\bar{b}\right)-2 n(\bar{a}+\bar{b})-1\right\} \eta(\mathcal{W}(\xi, U) Z) \\
+ & {\left[\left\{\frac{\bar{c} r}{2 n+1}\left(\frac{1}{2 n}+\bar{a}+\bar{b}\right)-2 n \bar{b}-1\right\}\left\{b r-\frac{2 n c r}{2 n+1}\left(\frac{1}{2 n}+a+b\right)\right\}\right.} \\
& \left.-\bar{a}\left\{b\|Q\|^{2}-\frac{c r^{2}}{2 n+1}\left(\frac{1}{2 n}+a+b\right)\right\}\right] g(Z, U) .
\end{aligned}
$$

$$
\begin{aligned}
& \sum_{i=1}^{2 n+1} g\left(\mathcal{W}\left(\omega\left(\xi, e_{i}\right) e_{i}, Z\right) U, \xi\right) \\
= & {\left[(2 n+1)\left\{1+2 n \bar{b}-\frac{\bar{c} r}{2 n+1}\left(\frac{1}{2 n}+\bar{a}+\bar{b}\right)\right\}+\bar{a} r\right] \eta(\mathcal{W}(\xi, U) Z) } \\
& +\left\{\frac{\bar{c} r}{2 n+1}\left(\frac{1}{2 n}+\bar{a}+\bar{b}\right)-2 n \bar{a}-1\right\} g\left(\mathcal{W}\left(e_{i}, Z\right) U, \xi\right) \eta\left(e_{i}\right) \\
& -\bar{b} g\left(\mathcal{W}\left(Q e_{i}, Z\right) U, \xi\right) \eta\left(e_{i}\right) \\
= & {\left[2 n\left\{1+2 n \bar{b}-\frac{\bar{c} r}{2 n+1}\left(\frac{1}{2 n}+\bar{a}+\bar{b}\right)\right\}+\bar{a} r+2 n \bar{b}-2 n \bar{a}\right] \eta(\mathcal{W}(\xi, U) Z) } \\
& +2 n \bar{b}\left[1+2 n b-\frac{c r}{2 n+1}\left(\frac{1}{2 n}+a+b\right)\right] g(Z, U)+2 n a \bar{b} S(Z, U) \\
& +2 n \bar{b}\left[1+2 n(a+b)-\frac{c r}{2 n+1}\left(\frac{1}{2 n}+a+b\right)\right] \eta(Z) \eta(U) .
\end{aligned}
$$




$$
\begin{aligned}
& \sum_{i=1}^{2 n+1} g\left(\mathcal{W}\left(e_{i}, \omega\left(\xi, e_{i}\right) Z\right) U, \xi\right) \\
& =\left\{1+2 n \bar{b}-\frac{\bar{c} r}{2 n+1}\left(\frac{1}{2 n}+\bar{a}+\bar{b}\right)\right\} g\left(e_{i}, Z\right) \eta\left(\mathcal{W}\left(e_{i}, \xi\right) U\right) \\
& \left\{\frac{\bar{c} r}{2 n+1}\left(\frac{1}{2 n}+\bar{a}+\bar{b}\right)-2 n \bar{a}-1\right\} \eta\left(\mathcal{W}\left(e_{i}, e_{i}\right) U\right) \eta(Z) \\
& +\bar{a} S\left(e_{i}, Z\right) \eta\left(\mathcal{W}\left(e_{i}, \xi\right) U\right)-\bar{b} \eta\left(\mathcal{W}\left(e_{i}, Q e_{i}\right) U\right) \eta(Z) \\
& =\left\{\frac{\bar{c} r}{2 n+1}\left(\frac{1}{2 n}+\bar{a}+\bar{b}\right)-2 n \bar{b}-1\right\} \eta(\mathcal{W}(\xi, U) Z) \\
& +2 n \bar{a}\left[1+2 n(a+b)-\frac{c r}{2 n+1}\left(\frac{1}{2 n}+a+b\right)\right] \eta(Z) \eta(U) \\
& +\bar{a}\left\{1+2 n b-\frac{c r}{2 n+1}\left(\frac{1}{2 n}+a+b\right)\right\} S(Z, U)+\bar{a} a S^{2}(Z, U) \\
& \sum_{i=1}^{2 n+1} g\left(\mathcal{W}\left(e_{i}, Z\right) \omega\left(\xi, e_{i}\right) U, \xi\right) \\
& =\left\{1+2 n \bar{b}-\frac{\bar{c} r}{2 n+1}\left(\frac{1}{2 n}+\bar{a}+\bar{b}\right)\right\} g\left(e_{i}, U\right) \eta\left(\mathcal{W}\left(e_{i}, Z\right) \xi\right) \\
& +\left\{\frac{\bar{c} r}{2 n+1}\left(\frac{1}{2 n}+\bar{a}+\bar{b}\right)-2 n \bar{a}-1\right\} \eta\left(\mathcal{W}\left(e_{i}, Z\right) e_{i}\right) \eta(U) \\
& +\bar{a} S\left(e_{i}, U\right) \eta\left(\mathcal{W}\left(e_{i}, Z\right) \xi\right) \\
& =\left\{\frac{\bar{c} r}{2 n+1}\left(\frac{1}{2 n}+\bar{a}+\bar{b}\right)-2 n \bar{a}-1\right\} \eta\left(\mathcal{W}\left(e_{i}, Z\right) e_{i}\right) \eta(U) \text {. }
\end{aligned}
$$

In view of (4.8), (4.16) reduces to

$$
\begin{aligned}
& \sum_{i=1}^{2 n+1} g\left(\mathcal{W}\left(e_{i}, Z\right) \omega\left(\xi, e_{i}\right) U, \xi\right) \\
= & -\left\{\frac{\bar{c} r}{2 n+1}\left(\frac{1}{2 n}+\bar{a}+\bar{b}\right)-2 n \bar{a}-1\right\}[2 n(1-a+2 n b) \\
+ & \left.\left\{a r-\frac{2 n c r}{2 n+1}\left(\frac{1}{2 n}+a+b\right)\right\}\right] \eta(U) \eta(Z) .
\end{aligned}
$$


By virtue of (4.13), (4.14), (4.15) \& (4.17), we obtain from (4.5) that

$$
\begin{aligned}
& {\left[\left\{\frac{\bar{c} r}{2 n+1}\left(\frac{1}{2 n}+\bar{a}+\bar{b}\right)-2 n \bar{b}-1+L\right\}(1+2 n a-b)\right.} \\
& -\bar{a}\left\{a r+\frac{c r}{2 n+1}\left(\frac{1}{2 n}+a+b\right)\right\}+a \bar{a} r \\
& +2 n a\left\{1-L+2 n \bar{b}-\frac{\bar{c} r}{2 n+1}\left(\frac{1}{2 n}+\bar{a}+\bar{b}\right)\right\} \\
& \left.-\bar{a}\left\{1+2 n b-\frac{c r}{2 n+1}\left(\frac{1}{2 n}+a+b\right)\right\}\right] S(Z, U) \\
& +\left[\left\{2 n(1-a+2 n b)+a r-\frac{2 n c r}{2 n+1}\left(\frac{1}{2 n}+a+b\right)\right\} \times\right. \\
& \left\{\frac{\bar{c} r}{2 n+1}\left(\frac{1}{2 n}+\bar{a}+\bar{b}\right)-2 n \bar{a}-1+L\right\} \\
& +\left\{\frac{c r}{2 n+1}\left(\frac{1}{2 n}+a+b\right)-2 n(a+b)-1\right\} \times \\
& \left\{2 n\left\{-\bar{b}+\frac{\bar{c} r}{2 n+1}\left(\frac{1}{2 n}+\bar{a}+\bar{b}\right)-1-2 n \bar{b}+L\right\}-\bar{a} r\right\} \\
& \left.-2 n(\bar{a}+\bar{b})\left\{1+2 n(a+b)-\frac{c r}{2 n+1}\left(\frac{1}{2 n}+a+b\right)\right\}\right] \eta(U) \eta(Z) \\
& +\left[\left\{\frac{\bar{c} r}{2 n+1}\left(\frac{1}{2 n}+\bar{a}+\bar{b}\right)-2 n \bar{b}-1+L\right\}\left\{b r-\frac{2 n c r}{2 n+1}\left(\frac{1}{2 n}+a+b\right)\right\}\right. \\
& +\left\{\frac{c r}{2 n+1}\left(\frac{1}{2 n}+a+b\right)-2 n b-1\right\} \\
& \left\{-2 n\left\{1-L+2 n \bar{b}-\frac{\bar{c} r}{2 n+1}\left(\frac{1}{2 n}+\bar{a}+\bar{b}\right)\right\}-\bar{a} r-2 n \bar{b}\right. \\
& \left\{-\bar{a}\left\{b\|Q\|^{2}-\frac{c r^{2}}{2 n+1}\left(\frac{1}{2 n}+a+b\right)\right\}\right. \\
& \left.-2 n \bar{b}\left\{1+2 n b-\frac{c r}{2 n+1}\left(\frac{1}{2 n}+a+b\right)\right\}\right] g(Z, U) \\
& +\bar{a}(b-1) S^{2}(Z, U)=0 \text {. }
\end{aligned}
$$

Theorem 4.1. Let $\left(M^{2 n+1}, g\right), n>1$ be an LP-Sasakian manifold. Then for respective pseudo symmetry type conditions, the Ricci tensor of the manifold $M$ takes the respective forms as follows- 


\begin{tabular}{|c|c|}
\hline $\begin{array}{l}\text { Curvature } \\
\text { condition }\end{array}$ & Expression for Ricci tensor \\
\hline $\begin{array}{c}R(X, Y) \cdot R= \\
L\{(X \wedge Y) \cdot R\} \\
(b y \bar{a}=\bar{b}=\bar{c}=0 \\
\& a=b=c=0)\end{array}$ & $\begin{array}{l}\text { For } L \neq 1 \\
S=2 n g\end{array}$ \\
\hline $\begin{array}{c}R(X, Y) \cdot C= \\
L\{(X \wedge Y) \cdot C\} \\
(b y \bar{a}=\bar{b}=\bar{c}=0, \\
\left.a=b=-\frac{1}{2 n-1} \& c=1\right) \\
\end{array}$ & $\begin{array}{l}\text { For } L \neq 1, \\
S=\left(\frac{r}{2 n}-1\right) g+\left(\frac{r}{2 n}-2 n-1\right) \eta \otimes \eta\end{array}$ \\
\hline $\begin{array}{c}R(X, Y) \cdot \hat{C}= \\
L\{(X \wedge Y) \cdot \hat{C}\} \\
(b y \bar{a}=\bar{b}=\bar{c}=0 \\
\left.a=b=-\frac{1}{2 n-1} \& c=0\right) \\
\end{array}$ & $\begin{array}{l}\text { For } L \neq 1 \\
S=\left(\frac{r}{2 n}-1\right) g+\left(\frac{r}{2 n}-2 n-1\right) \eta \otimes \eta\end{array}$ \\
\hline $\begin{array}{c}R(X, Y) \cdot E= \\
L\{(X \wedge Y) \cdot E\} \\
(b y \bar{a}=\bar{b}=\bar{c}=0 \\
a=b=0 \& c=1)\end{array}$ & $\begin{array}{l}\text { For } L \neq 1 \\
S=2 n g\end{array}$ \\
\hline $\begin{array}{c}R(X, Y) \cdot P= \\
L\{(X \wedge Y) \cdot P\} \\
(b y \bar{a}=\bar{b}=\bar{c}=0, \\
\left.a=-\frac{1}{2 n} \& b=c=0\right) \\
\end{array}$ & $\begin{array}{l}\text { For } L \neq 1 \\
S=2 n g+\left(\frac{r}{2 n}-2 n-1\right) \eta \otimes \eta .\end{array}$ \\
\hline $\begin{array}{c}R(X, Y) \cdot H= \\
L\{(X \wedge Y) \cdot H\} \\
(b y \bar{a}=\bar{b}=\bar{c}=0 \\
\left.a=b=-\frac{1}{4 n} \& c=0\right)\end{array}$ & $\begin{array}{l}\text { For } L \neq 1 \\
S=\left(\frac{r+4 n^{2}}{4 n+1}\right) g+\left\{\frac{r-2 n(2 n+1)}{4 n+1}\right\} \eta \otimes \eta\end{array}$ \\
\hline $\begin{array}{c}E(X, Y) \cdot R= \\
L\{(X \wedge Y) \cdot R\} \\
(b y \bar{a}=\bar{b}=0, \bar{c}=1 \\
\& a=b=c=0)\end{array}$ & $\begin{array}{l}\text { For } L \neq 1-\frac{r}{2 n(2 n+1)} \\
S=2 n g\end{array}$ \\
\hline $\begin{array}{c}E(X, Y) \cdot C= \\
L\{(X \wedge Y) \cdot C\} \\
(b y \bar{a}=0=\bar{b}, \bar{c}=1 \\
\left.a=b=-\frac{1}{2 n-1}, c=1\right) \\
\end{array}$ & $\begin{array}{l}\text { For } L \neq 1-\frac{r}{2 n(2 n+1)}, \\
S=\left(\frac{r}{2 n}-1\right) g+\left(\frac{r}{2 n}-2 n-1\right) \eta \otimes \eta\end{array}$ \\
\hline $\begin{array}{c}E(X, Y) \cdot \hat{C}= \\
L\{(X \wedge Y) \cdot \hat{C}\} \\
(b y \bar{a}=0=\bar{b}, \bar{c}=1 \\
\left.a=b=-\frac{1}{2 n-1} \& c=1\right)\end{array}$ & $\begin{array}{l}\text { For } L \neq 1-\frac{r}{2 n(2 n+1)}, \\
S=\left(\frac{r}{2 n}-1\right) g+\left(\frac{r}{2 n}-2 n-1\right) \eta \otimes \eta\end{array}$ \\
\hline
\end{tabular}




\begin{tabular}{|c|c|}
\hline $\begin{array}{l}\text { Curvature } \\
\text { condition }\end{array}$ & Expression for Ricci tensor \\
\hline $\begin{array}{c}E(X, Y) \cdot E= \\
L\{(X \wedge Y) \cdot E\} \\
(b y \bar{a}=0=\bar{b}, \bar{c}=1 \\
a=b=0 \& c=1)\end{array}$ & $\begin{array}{l}\text { For } L \neq 1-\frac{r}{2 n(2 n+1)} \\
S=2 n g\end{array}$ \\
\hline $\begin{array}{c}E(X, Y) \cdot P= \\
L\{(X \wedge Y) \cdot P\} \\
(b y \bar{a}=0=\bar{b}, \bar{c}=1 \\
\left.a=-\frac{1}{2 n}, b=0 \& c=1\right)\end{array}$ & $\begin{array}{l}\text { For } L \neq 1-\frac{r}{2 n(2 n+1)} \\
S=2 n g+\left(\frac{r}{2 n}-2 n-1\right) \eta \otimes \eta\end{array}$ \\
\hline $\begin{array}{c}E(X, Y) \cdot H= \\
L\{(X \wedge Y) \cdot H\} \\
(b y \bar{a}=0=\bar{b}, \bar{c}=1 \\
\left.a=b=-\frac{1}{4 n} \& c=1\right)\end{array}$ & $\begin{array}{l}\text { For } L \neq 1-\frac{r}{2 n(2 n+1)} \\
S=\left(\frac{r+4 n^{2}}{4 n+1}\right) g+\left\{\frac{r-2 n(2 n+1)}{4 n+1}\right\} \eta \otimes \eta\end{array}$ \\
\hline $\begin{array}{c}\hat{C}(X, Y) \cdot R= \\
L\{(X \wedge Y) \cdot R\} \\
\left(b y \bar{a}=\bar{b}=-\frac{1}{2 n-1}, \bar{c}=0\right. \\
\& a=b=c=0)\end{array}$ & $\begin{array}{l}\{2+(2 n-1) L\} S=\{r+2 n+2 n(2 n-1) L\} g \\
+(r-2 n) \eta \otimes \eta+S^{2} .\end{array}$ \\
\hline $\begin{array}{c}\hat{\hat{C}}(X, Y) \cdot \hat{C}= \\
L\{(X \wedge Y) \cdot \hat{C}\} \\
\left(b y \bar{a}=\bar{b}=-\frac{1}{2 n-1}, \bar{c}=0\right. \\
\left.a=b=-\frac{1}{2 n-1} \& c=0\right) \\
\end{array}$ & $\begin{array}{l}(2 n-1)(1+2 n L) S=2 n S^{2} \\
+\left\{\left(\|Q\|^{2}-2 n\right)-(2 n-1)(2 n-r) L\right\} g \\
+[(2 n-1)\{r-2 n(2 n+1)\} L-2 n r] \eta \otimes \eta\end{array}$ \\
\hline $\begin{array}{c}\hat{C}(X, Y) \cdot C= \\
L\{(X \wedge Y) \cdot C\} \\
\left(b y \bar{a}=\bar{b}=-\frac{1}{2 n-1}, \bar{c}=0\right. \\
\left.a=b=-\frac{1}{2 n-1} \& c=1\right) \\
\end{array}$ & $\begin{array}{l}(2 n-1)(1+2 n L) S \\
=\left\{2 n L(2 n-1)\left(\frac{r}{2 n}-1\right)+\|Q\|^{2}-2 n\right\} g \\
+\{r+2 n L(2 n-1)\}\left\{\frac{r}{2 n}-(2 n+1)\right\} \eta \otimes \eta \\
-2 n S^{2}\end{array}$ \\
\hline $\begin{array}{c}\hat{C}(X, Y) \cdot E= \\
L\{(X \wedge Y) \cdot E\} \\
\left(b y \bar{a}=\bar{b}=-\frac{1}{2 n-1}, \bar{c}=0\right. \\
\& a=b=c=0) \\
\quad \& a\end{array}$ & $\begin{array}{l}\{2+(2 n-1) L\} S=-S^{2} \\
+[r+2 n+2 n(2 n-1) L] g \\
+(2 n-r)\left\{\frac{r}{2 n(2 n+1)}-1\right\} \eta \otimes \eta\end{array}$ \\
\hline $\begin{array}{c}\hat{C}(X, Y) \cdot P= \\
L\{(X \wedge Y) \cdot P\} \\
\left(b y \bar{a}=\bar{b}=-\frac{1}{2 n-1}, \bar{c}=0\right. \\
\left.a=-\frac{1}{2 n}, b=0 \& c=0\right)\end{array}$ & $\begin{array}{l}\{2+(2 n-1) L\} S=-S^{2} \\
+[r+2 n+2 n(2 n-1) L] g \\
+\left(\frac{r}{2 n}-2 n-1\right)\{1+(2 n-1) L\} \eta \otimes \eta\end{array}$ \\
\hline $\begin{array}{c}\hat{C}(X, Y) \cdot H= \\
L\{(X \wedge Y) \cdot H\} \\
\left(b y=\bar{a}=\bar{b}=-\frac{1}{2 n-1}, \bar{c}=0\right. \\
\left.a=b=-\frac{1}{4 n} \& c=0\right)\end{array}$ & $\begin{array}{l}\frac{\{1+(2 n-1) L\}(4 n+1)+2 n}{4 n} S \\
=\left[\left(\frac{r}{4 n}+n\right)\{1+(2 n-1) L\}\right. \\
\left.+\frac{r}{2}+\frac{1}{4 n}\|Q\|^{2}\right] g-\left(1+\frac{1}{4 n}\right) S^{2} \\
+\left(\frac{r}{4 n}-\frac{2 n+1}{2}\right)\{1+(2 n-1) L\} \eta \otimes \eta\end{array}$ \\
\hline
\end{tabular}




\begin{tabular}{|c|c|}
\hline $\begin{array}{l}\text { Curvature } \\
\text { condition }\end{array}$ & Expression for Ricci tensor \\
\hline $\begin{array}{c}P(X, Y) \cdot R= \\
L\{(X \wedge Y) \cdot R\} \\
\left(b y \bar{a}=-\frac{1}{2 n}, \bar{b}=0, \bar{c}=0\right. \\
\& a=b=c=0)\end{array}$ & $\begin{array}{l}\left(\frac{1}{2 n}-1+L\right) S=\left(\frac{r}{2 n}-2 n+2 n L\right) g \\
+\left(\frac{r}{2 n}-2 n-1\right) \eta \otimes \eta-\frac{1}{2 n} S^{2} .\end{array}$ \\
\hline $\begin{array}{c}P(X, Y) \cdot \hat{C}= \\
L\{(X \wedge Y) \cdot \hat{C}\} \\
\left(b y \bar{a}=-\frac{1}{2 n}, \bar{b}=0, \bar{c}=0\right. \\
\left.a=b=-\frac{1}{2 n-1} \& c=0\right)\end{array}$ & $\begin{array}{l}\frac{4 n^{2}+1-4 n^{2} L}{2 n} S \\
=\left\{\frac{2 n+1}{2 n} r-2 n-\frac{\|Q\|^{2}}{2 n}+(2 n-L) r\right\} g \\
+\left\{\frac{2 n+1}{2 n} r-(2 n+1)^{2}-r L\right\} \eta \otimes \eta+S^{2} .\end{array}$ \\
\hline $\begin{array}{l}P(X, Y) \cdot C=\quad \text { Obtain } \\
L\{(X \wedge Y) \cdot C\} \quad \text { O } \\
b y \bar{a}=-\frac{1}{2 n}, \bar{b}=0, \bar{c}=0 \\
\left.a=b=-\frac{1}{2 n-1} \& c=1\right)\end{array}$ & $\begin{array}{l}\frac{4 n^{2}(L-1)-1}{2 n} S \\
=\left\{\begin{array}{c}\left(\frac{r}{2 n}-2 n+2 n L\right)\left(\frac{r}{2 n}-1\right) \\
-\frac{1}{2 n}\left(\frac{r^{2}}{2 n}-\|Q\|^{2}\right)\end{array}\right\} g \\
+\left\{\frac{r}{2 n}-(2 n+1)\right\}\left\{\frac{r}{2 n}-(2 n+1)+2 n L\right\} \eta \otimes \eta \\
-S^{2} .\end{array}$ \\
\hline $\begin{array}{c}P(X, Y) \cdot E= \\
L\{(X \wedge Y) \cdot E\} \\
\left(b y \bar{a}=-\frac{1}{2 n}, \bar{b}=0, \bar{c}=0\right. \\
a=b=0 \& c=1)\end{array}$ & $\begin{array}{l}\frac{4 n^{2}(L-1)-1}{2 n} S \\
=\left\{\begin{array}{c}\left(\frac{r}{2 n}-2 n+2 n L\right)\left(\frac{r}{2 n}-1\right) \\
-\frac{1}{2 n}\left(\frac{r^{2}}{2 n}-\|Q\|^{2}\right)\end{array}\right\} g \\
+\left\{\frac{r}{2 n}-(2 n+1)\right\}\left\{\frac{r}{2 n}-(2 n+1)+2 n L\right\} \eta \otimes \eta \\
-S^{2} .\end{array}$ \\
\hline $\begin{array}{c}P(X, Y) \cdot P= \\
L\{(X \wedge Y) \cdot P\} \\
\left(b y \bar{a}=-\frac{1}{2 n}, \bar{b}=0, \bar{c}=0\right. \\
\left.a=-\frac{1}{2 n}, b=0 \& c=0\right)\end{array}$ & $\begin{array}{l}(1-2 n+2 n L) S=\left(r-4 n^{2}+4 n^{2} L\right) g \\
+L\{r-2 n(2 n+1)\} \eta \otimes \eta-S^{2} .\end{array}$ \\
\hline $\begin{array}{c}P(X, Y) \cdot H= \\
L\{(X \wedge Y) \cdot H\} \\
\left(b y \bar{a}=-\frac{1}{2 n}, \bar{b}=0, \bar{c}=0\right. \\
\left.a=b=-\frac{1}{4 n} \& c=0\right)\end{array}$ & $\begin{array}{l}\frac{4 n-(4 n+1) L}{4 n} S=\frac{1}{2 n}\left(1+\frac{1}{4 n}\right) S^{2} \\
+\left\{n-\frac{1}{8 n^{2}}\|Q\|^{2}-\left(\frac{r}{4 n}+n\right) L\right\} g+ \\
+\frac{1}{4 n} L\{2 n(2 n+1)-r\} \eta \otimes \eta\end{array}$ \\
\hline $\begin{array}{c}H(X, Y) \cdot R= \\
L\{(X \wedge Y) \cdot R\} \\
\text { by } \bar{a}=\bar{b}=-\frac{1}{4 n}, \bar{c}=0 \\
\quad \& a=b=c=0) \\
\end{array}$ & $\begin{array}{c}\left\{\frac{1-2 n}{4 n}+L\right\} S=\left(\frac{r}{4 n}-n+2 n L\right) g \\
+\left(\frac{r}{4 n}-\frac{1}{2}\right) \eta \otimes \eta-\frac{1}{4 n} S^{2} .\end{array}$ \\
\hline $\begin{array}{c}H(X, Y) \cdot \hat{C}= \\
L\{(X \wedge Y) \cdot \hat{C}\} \\
b y \bar{a}=\bar{b}=-\frac{1}{4 n}, \bar{c}=0 \\
\left.a=b=-\frac{1}{2 n-1} \& c=0\right)\end{array}$ & $\begin{array}{l}\left(\frac{4 n^{2}+1}{4 n}-2 n L\right) S=\frac{1}{2} S^{2} \\
+\left\{\frac{2 n+1}{4 n} r-n-\frac{\|Q\|^{2}}{4 n}+(2 n-r) L\right\} g \\
+\left[\frac{4 n+1}{4 n} r-\frac{(2 n+1)^{2}}{2}+\{2 n(2 n+1)-r\} L\right] \eta \otimes \eta .\end{array}$ \\
\hline $\begin{array}{c}H(X, Y) \cdot E= \\
L\{(X \wedge Y) \cdot E\} \\
b y \bar{a}=\bar{b}=-\frac{1}{4 n},, \bar{c}=0 \\
a=b=0 \& c=1)\end{array}$ & $\begin{array}{l}\left(\frac{1-2 n}{4 n}+L\right) S=\left(\frac{r}{4 n}-n+2 n L\right) g \\
+\left(\frac{r}{2 n}-1\right)\left\{-\frac{r}{4 n(2 n+1)}+\frac{1}{2}\right\} \eta \otimes \eta-\frac{1}{4 n} S^{2} .\end{array}$ \\
\hline
\end{tabular}




\begin{tabular}{|c|c|}
\hline $\begin{array}{l}\text { Curvature } \\
\text { condition }\end{array}$ & Expression for Ricci tensor \\
\hline $\begin{array}{c}H(X, Y) \cdot P= \\
L\{(X \wedge Y) \cdot P\} \\
b y \bar{a}=\bar{b}=-\frac{1}{4 n}, \bar{c}=0 \\
\left.a=-\frac{1}{2 n}, b=c=0\right) \\
\end{array}$ & $\begin{array}{l}\frac{1-2 n}{4 n} S=\left(\frac{r}{4 n}-n+2 n L\right) g \\
+\left\{-\frac{r}{4 n}+\frac{2 n+1}{2}\right\}(1-2 L) \eta \otimes \eta \\
-\frac{1}{4 n} S^{2}\end{array}$ \\
\hline $\begin{array}{c}H(X, Y) \cdot H= \\
L\{(X \wedge Y) \cdot H\} \\
b y \bar{a}=\bar{b}=-\frac{1}{4 n}, \bar{c}=0 \\
\left.a=b=-\frac{1}{4 n} \& c=0\right)\end{array}$ & $\begin{array}{l}S=\left\{\left(\frac{2 n+1}{2}-\frac{1}{8 n^{2}}\|Q\|^{2}\right)-\right. \\
\left.\left(\frac{r}{2 n}+2 n\right) L\right\} g+\frac{1}{2 n}\left(1+\frac{1}{4 n}\right) S^{2} \\
+(1-2 L)\left(\frac{r}{4 n}-\frac{2 n+1}{2}\right) \eta \otimes \eta\end{array}$ \\
\hline $\begin{array}{c}C(X, Y) \cdot R= \\
L\{(X \wedge Y) \cdot R\} \\
\left(b y \bar{a}=\bar{b}=-\frac{1}{2 n-1}, \bar{c}=1\right. \\
a=b=c=0)\end{array}$ & $\begin{array}{l}\left\{\left(2-\frac{r}{2 n}\right)+(2 n-1) L\right\} S \\
=\{2 n+2 n(2 n-1) L\} g \\
+\{r-2 n-4 n(n-1) L\} \eta \otimes \eta-S^{2} .\end{array}$ \\
\hline $\begin{array}{c}C(X, Y) \cdot \hat{C}= \\
L\{(X \wedge Y) \cdot \hat{C}\} \\
b y \bar{a}=\bar{b}=-\frac{1}{2 n-1}, \bar{c}=1 \\
\left.a=b=-\frac{1}{2 n-1}, c=0\right)\end{array}$ & $\begin{array}{l}\{2 n-1-r+2 n(2 n-1) L\} S \\
=\left\{\left(r-\frac{r^{2}}{2 n}\right)-2 n-(2 n-r) L\right. \\
\left.+\|Q\|^{2}\right\} g+\left[\left(r-\frac{r^{2}}{2 n}\right)-(2 n-1) \times\right. \\
\{2 n(2 n+1)-r\} L] \eta \otimes \eta-2 n S^{2} .\end{array}$ \\
\hline $\begin{array}{c}C(X, Y) \cdot P= \\
L\{(X \wedge Y) \cdot P\} \\
\left(b y \bar{a}=\bar{b}=-\frac{1}{2 n-1}, \bar{c}=1\right. \\
\left.a=-\frac{1}{2 n}, b=c=0\right) \\
\end{array}$ & $\begin{array}{l}\left\{\left(2-\frac{r}{2 n}\right)+(2 n-1) L\right\} S= \\
\{2 n+2 n(2 n-1) L\} g \\
+\left(\frac{r}{2 n}-1-(2 n-1) L\right) \times \\
\left(2 n+1-\frac{r}{2 n}\right) \eta \otimes \eta-S^{2}\end{array}$ \\
\hline $\begin{array}{c}C(X, Y) \cdot E= \\
L\{(X \wedge Y) \cdot E\} \\
\left(b y \bar{a}=\bar{b}=-\frac{1}{2 n-1}, \bar{c}=1\right. \\
a=b=0, c=1)\end{array}$ & $\begin{array}{l}\left\{\left(2-\frac{r}{2 n}\right)+(2 n-1) L\right\} S=2 n g \\
+\left\{\begin{array}{c}\left(\frac{r}{2 n}-1\right)\left(2 n-\frac{r}{2 n+1}\right)+ \\
(1-n)\left(4 n-\frac{2 r}{2 n+1}\right) L\end{array}\right\} \eta \otimes \eta \\
-S^{2}\end{array}$ \\
\hline $\begin{array}{c}C(X, Y) \cdot H= \\
L\{(X \wedge Y) \cdot H\} \\
\left(b y \bar{a}=\bar{b}=-\frac{1}{2 n-1}, \bar{c}=1\right. \\
\left.a=b=-\frac{1}{4 n}, c=0\right)\end{array}$ & 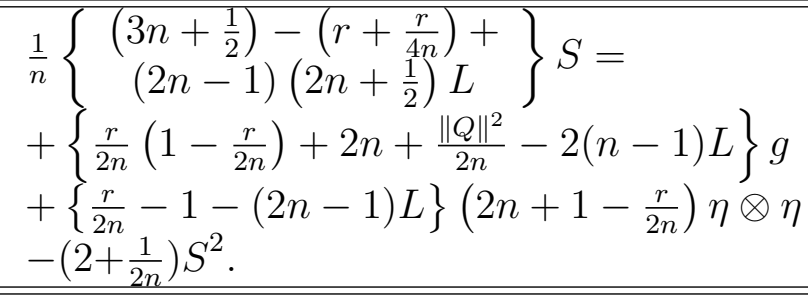 \\
\hline
\end{tabular}

Remark 4.1. For $L=0$, the above theorem gives us the nature of the Ricci tensor of the manifold $M$ for respective semi-symmetry type conditions. 


\section{References}

[1] A. A. Shaikh and K. K. Baishya, On $\phi$-symmetric LP-Sasakian manifolds, Yokohama Math. J., 52, (2005), 97-112.

[2] A. A. Shaikh and K. K. Baishya, Some results on LP-Sasakian manifolds, Bull. Math. Soc. Sc. Math. Rommanic, Tome 49(97), (2006), 197-208.

[3] A. A. Shaikh, T. Basu, and K. K. Baishya, On the exixtence of locally $\phi$ recurrent LP-Sasakian manifolds, Bull. Allahabad Math. Soc., 24(2), (2009), 281-295.

[4] B. J. Papantoniou, Contact Riemannian manifolds satisfying $R(\xi, X) \cdot R=0$ and $\xi \in(k, \mu)$-nullity distribution, Yokohama Math. J., 40, (1993), 149-161.

[5] D. Perrone, Contact Riemannian manifolds satisfying $R(\xi, X) \cdot R=0$, Yokohama Math. J., 39(2), (1992), 141-149.

[6] E. Cartan, Sur une classe remarquable d'espaces de Riemannian, Bull. Soc. Math. France, 54, (1926), 214-264.

[7] E. Boeckx, O. Kowalski, and L. Vanhecke, Riemannian Manifolds of Conullity Two, World Scientific Publishing Co., Inc., River Edge, NJ, 1996.

[8] G. Calvaruso and D. Perrone, Semi-symmetric contact metric three-manifolds, Yokohama Math. J., 49(2), (2002), 149-161.

[9] F. Defever and R. Deszcz, On semi-Riemannian manifolds satisfying the condition $R \cdot R=Q(S, R)$, World Scientific, Singapore, (1991), 108-130.

[10] F. Defever and R. Deszcz, On warped product manifolds satisfying a certain curvature condition, Atti. Acad. Peloritana Pericolanti Cl. Sci. Fis. Mat. Natur., 69, (1991), 213-236.

[11] F. Gouli-Andreou and E. Moutafi, Two classes of pseudosymmetric contact metric 3-manifolds, Pacific J. Math., 239(1), (2009), 17-37.

[12] F. Gouli-Andreou and E. Moutafi, Three classes of pseudosymmetric contact metric 3-manifolds, Pacific J. Math., 245(1), (2010), 57-77.

[13] G. P. Pokhariyal and R.S. Mishra, Curvatur tensors' and their relativistics significance I, Yokohama Math. J., 18, (1970), 105-108.

[14] K. K. Baishya and P. R. Chowdhury, On generalized quasi-conformal $N(k, \mu)$ manifolds, Commun. Korean Math. Soc., 31(1), (2016), 163-176.

[15] K. Nomizu, On the decomposition of generalized curvature tensor fields, Differential Geometry in honor of K. Yano, (1972), 335-345.

[16] K. Matsumoto, On Lorentzian almost paracontact manifolds, Bull. of Yamagata Univ. Nat. Sci., 12, (1989), 151-156.

[17] K. Sekigawa, On 4-dimensional connected Einstein spaces satisfying the condition $R(X, Y) \cdot R=0$, Sci. Rep. Nigaata Univ. Ser. A, 7, (1969), 29-31.

[18] K. Yano and S. Bochner, Curvature and Betti numbers, Annals of Math. Studies, 32, (1953.) 
[19] K. Yano and S. Sawaki, Riemannian manifolds admitting a conformal transformation group, J. Diff. Geom., 2, (1968), 161-184.

[20] I. Mihai and R. Rosca, On Lorentzian P-Sasakian manifolds, Classical Analysis, World Scientific Publi., Singapore, (1992), 155-169.

[21] L. P.Eisenhart, Riemannian Geometry, Princeton University Press, 1949.

[22] M. Belkhelfa, Differential geometry of semi-Riemannian manifolds and submanifolds, Ph.D. thesis, K. U. Leuven, 2001.

[23] M. Belkhelfa, R. Deszcz, and L. Verstraelen, Symmetry properties of Sasakian space forms, Soochow J. Math., 31, (2005), 611-616.

[24] M. C. Chaki, On pseudo-symmetric manifolds, Ann. Stiint. Univ. Al.I. Cuza Iaşi Sect. I-a Math. N.S., 33, (1987), 53-58.

[25] M. Tarafdar, On pseudo-symmetric and pseudo-Ricci-symmetric Sasakian manifolds, Period. Math. Hung., 22, (1991), 125-128.

[26] N.S. Sinjukov, On geodesic maps of Riemannian space, Matem. Sjezda, (1956), 167-168.

[27] O. Kowalski, An explicit classification of three dimensional Riemannian spaces satisfying $R(X, Y) \cdot R=0$, Czechoslovak Math. J., 46(121), (1996), 427-474.

[28] O. Kowalski and M. Sekizawa, Three-dimensional Riemannian manifolds of cconullity two, World Scientific, Singapore, Chapter 11, (1996.)

[29] R. Deszcz, On pseudo-symmetric spaces, Bull. Soc. Math. Belg. Ser. A, 44, (1992), $1-34$

[30] R. Deszcz, On four-dimensional warped product manifolds satisfying certain pseudosymmetry curvature conditions, 62, (1991), 103-120.

[31] R. Deszcz, On Ricci-pseudo symmetric warped products, Demonstratio Math., 22, (1989), 1053-1065.

[32] R. Deszcz and W. Grycak, On some class of warped product manifolds, Bull. Inst. Math. Acad. Sinica., 15, (1987), 311-322.

[33] R. Deszcz, S. Haesen, and L. Verstraelen, On natural symmetries, Topics in Differential Geometry, Ch.6, Editors A. Mihai, I. Mihai and R. Miron, Editura Academiei Române, 2008.

[34] R. Deszcz and M. Hotloś, On some pseudo-symmetry type curvature condition, Tsukuba J. Math., 27, (2003), 13-30.

[35] S. Haesen and L. Verstraelen, Natural intrinsic geometrical symmetries, Symmetry, Integrability and Geometry: Methods and Applications, (SIGMA) 5, 086, 14 pp. Special Issue Elie Cartan and Differential Geometry, 2009.

[36] S. Sasaki, Lecture Notes on Almost Contact Manifolds, Part I, Tohoku Univ, 1965.

[37] U. C. De, K. Matsumoto, and A. A. Shaikh, On Lorentzian para-Sasakian manifolds, Rendiconti del Seminario Mat. de Messina, 3, (1999), 149-156.

[38] Y.Ishii, On conharmonic transformations, Tensor(N.S.), 7, (1957), 73-80. 
Vol. LIV (2016) Deszcz pseudo symmetry type LP-Sasakian manifolds

[39] Z. I. Szabö, Classification and construction of complete hypersurfaces satisfying $R(X, Y) \cdot R=0$. , Acta. Sci. Math., 47, (1984), 321-348.

[40] Z. I. Szabö, Structure theorems on Riemannian spaces satisfying $R(X, Y) \cdot R=0$, J. Differential Geom, 17: I, The local version, (1982), 531-582.

[41] Z. I. Szabö, Geom. Dedicata, 19: II, Global version, (1985), 65-108.

Kanak Kanti Baishya

Department Of Mathematics,

Kurseong College, Dowhill Road, Kurseong,

Darjeeling-734203, West Bengal, India

E-mail: kanakkanti.kc@gmail.com

Partha Roy Chowdhury

Department Of Mathematics,

Shaktigarh Bidyapith(H.S), Siliguri,

Darjeeling-734005, West Bengal, India

E-mail: partha.raychowdhury81@gmail.com

Received: 3.02 .2016

Accepted: 14.07.2016 